\title{
Comparison among the Short-Term Effect of Massage Therapy, Central Stability Exercise and Combination Method on Limits of Stability in Patients with Chronic Non-Specific Low Back Pain
}

\section{ARTICLE INFO}

\section{Article Type}

Original Research

\section{Authors}

Shakeri A.* $M S c$

Sokhangoei $Y{ }^{1} P h D$,

Hoseini $Y^{2} P h D$

How to cite this article
Shakeri A, Sokhangoei Y, Hoseini Y.
Comparison among the Short-
Term Effect of Massage Therapy,
Central Stability Exercise and
Combination Method on Limits of
Stability in Patients with Chronic
Non-Specific Low Back Pain.
Horizon of Medical Sciences.
2017;23(1):13-19.

*Physical Education Department, Physical Education \& Sports Sciences Faculty, Kharazmi University, Tehran, Iran

${ }^{1}$ Physiotherapy Department, Physiotherapy Faculty, University of Social Welfare and Rehabilitation Sciences, Tehran, Iran

${ }^{2}$ Physical Education Department, Physical Education \& Sports Sciences Faculty, Scientific-Applied University of Physical Education, Hamedan, Iran

\section{Correspondence}

Address: No. 49, Shahid Mofateh Street, Tehran, Iran. Postal Code: 15719-14911

Phone: +98 (21) 77299848

Fax: +98 (21) 77292669

alishakeri310@gmail.com

\section{Article History}

Received: November 20, 2015

Accepted: July 19, 2016

ePublished: January 19, 2017

\section{A B S T RACT}

Aims Nowadays, balance and postural control index is assessed as one of the main parameters in the assessment of persons with neuromuscular and musculoskeletal disorders. The aim of the study was to investigate the short term effects of massage therapy, core stability exercises and combined stability exercises on the stability limits in patients with chronic non-specific low back pain.

Materials \& Methods In the semi-experimental study, 30 male patients with chronic nonspecific LBP, referred to the physiotherapy clinics of 1 and 3 districts of Tehran City, were studied in 2013. The subjects were selected via random sampling method, were randomly divided into three groups including massage therapy, core stability exercise, and combined stability exercises. 12-session treatment protocols (4 weeks) were conducted in the groups under the therapist's supervision. The force plate device was used to measure the reaction time (RT), movement velocity (MV), maximum excursion (ME), and end point of excursion (EPE) in eight different directions. Data was analyzed by SPSS 16 software using ANCOVA test. Findings After the treatment interventions, the combined exercises improved RT at directions 3 (right lateral) and 8 (left anterior) more than the stability exercises and massage therapy. In addition, more improvement in MV was recorded at the direction 2 (right anterior) compared to the stability exercises. ME records of stability and combined exercises groups were significantly different at direction 7 (left lateral) only $(\mathrm{p}<0.05)$.

Conclusion Despite the fact that each treatment intervention has short term effects on the stability limits of patients with chronic non-specific LBP, it seems that the combination of core stability exercises and massage therapy is more effective than each one solely.

Keywords Massage; Low Back Pain; Exercise Therapy; Core Stability Exercises; Stability Limits

\section{CIT A T I O N L INKS}

[1] Epidemiology and risk factors for spine ... [2] Low back pain in Australian ... [3] Spinal segmental stabilization exercises combined withtraditional strengthening exercise program in ... [4] Massage for low back pain: An updated systematic review within the framework of the Cochrane Back Review ... [5] A systematic review of efficacy of McKenzie therapy for spinal ... [6] The effects of stability training on postural indices and limit of stability in male patients with nonspecific chronic low ... [7] Physiology of the ... [8] Therapeutic exercise: Foundations and ... [9] Evaluation of postural balance using the biodex balance system in subjects with and without low ... [10] Muscle testing and ... [11] Pathophysiological model for chronic low back pain integrating connective tissue and nervous system ... [12] Muscle imbalance and the low ... [13] Core stability exercise ... [14] Techniques for active lumbar stabilisation for spinal protection: a pilot ... [15] Diagnosis and classification of chronic low back ... [16] Muscle strength in relation to muscle length, pain and muscle ... [17] Muscles, central nervous motor regulation and back ... [18] Exercise therapy for low back pain: Asmall-scale exploratory survey of current physiotherapy practice in the Republic of Irland acute hospital ... [19] Sensory-motor control of the lower back: Implications for ... [20] Effects of two 4-week proprioceptive ... [21] Muscle activation during exercises to ... [22] Persistence of improvements in postural strategies following motor control ... [23] Motor training of the lumbar paraspinal muscles induces immediate changes in motor coordination in patients with recurrent low back ... [24] The effects of consecutive supervised stability training on postural balance in ... [25] Stabilisation exercises for low ... [26] Evaluation of dynamic balance ... [27] Generalizability of the limits of stability test in ... [28] Compared massage and modalities in the ... [29] Quantitative evaluation of ... [30] The effects of stability exercise ... [31] Impaired postural control of ... 
علاوه بر اين، نتايج تحقيقات اخير نشان داده است كه در افراد

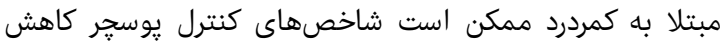

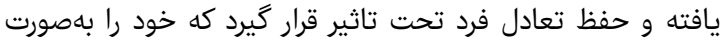

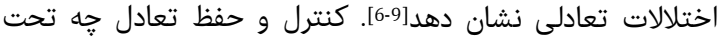

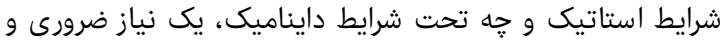

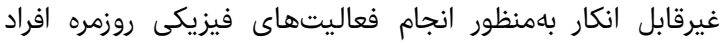

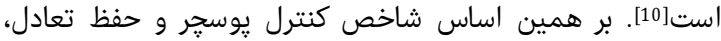

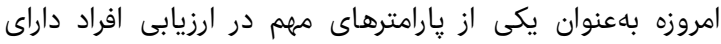

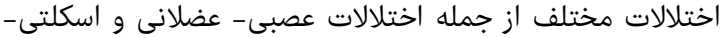

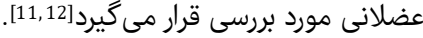

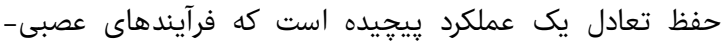

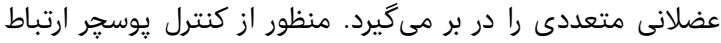

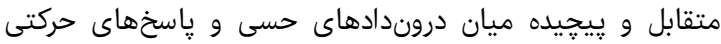

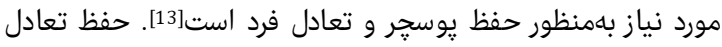

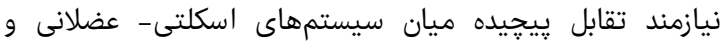

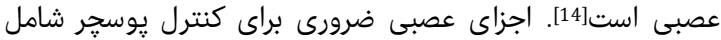

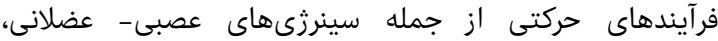

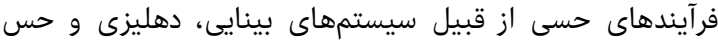

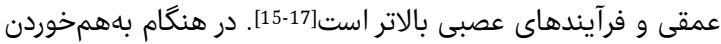

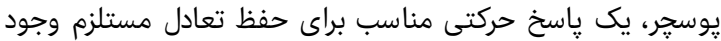

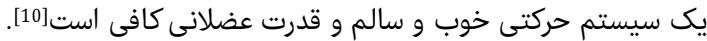

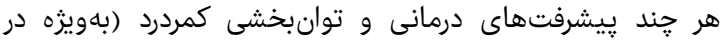

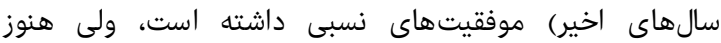

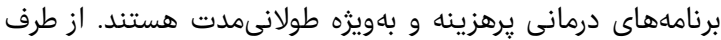

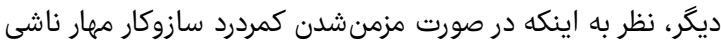

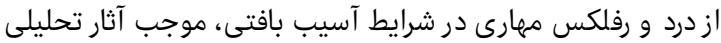

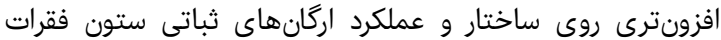

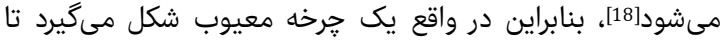

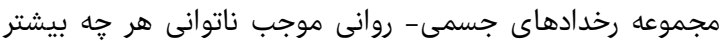

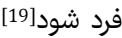

تمريندرمانى يكى از رايجترين مداليتههاى درمانى براى بردئ بيماران

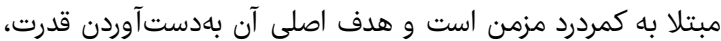

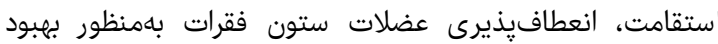

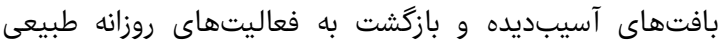

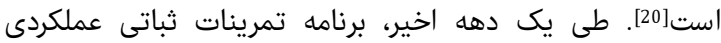

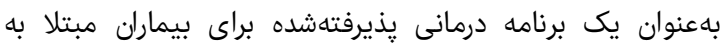

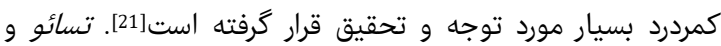

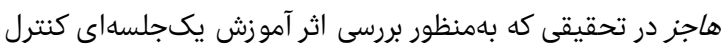

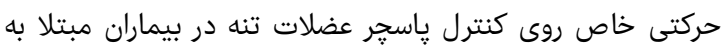

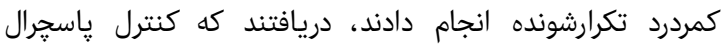

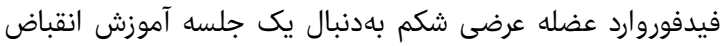

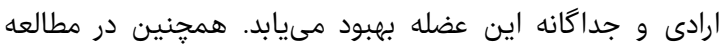

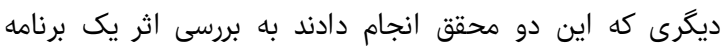

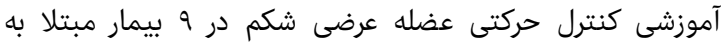

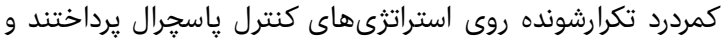

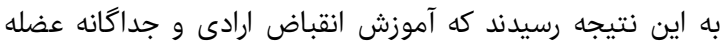

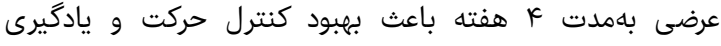

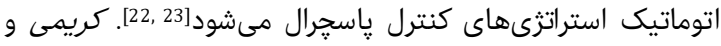

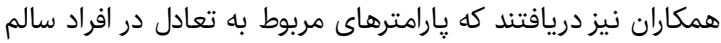

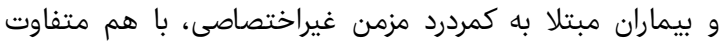

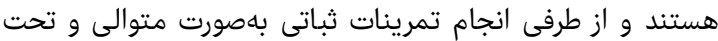

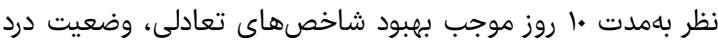
و توانايى عملكردى اين بيماران مى شود[2]ـ.
مقايسه تاثير كوتاهمدت ماساردرمانى، تمرينات

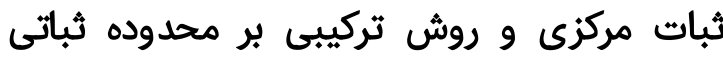

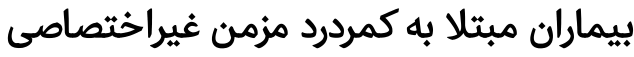

MSc "على شاكرى

كرَوه تربيت بدنى، داكري دانشكده تربيت بدنى و علوم ورزشى، دانشكاه خوارزمى،

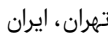

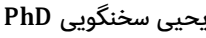
كروه فيزيوترإيى، دانشكده فيزيوترايى، دانشكاه علوم بهزيستى و توانبخشى، تيهران، ايران

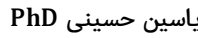
كرّوه تربيت بدنى، دانشكده تربيت بدنى و علوم ورزشى، دانشكاه علمى - كاربردى تربيت بدنى، همدان، ايران

קكيده

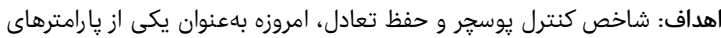

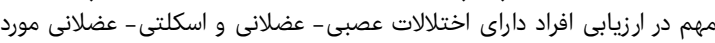

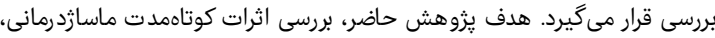

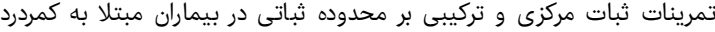

مزمن غيراختصاصى بود. ت مورين

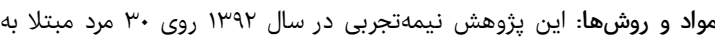

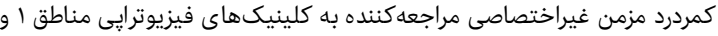

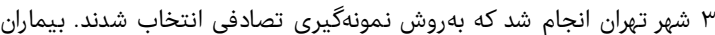

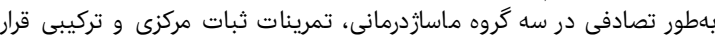

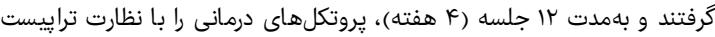

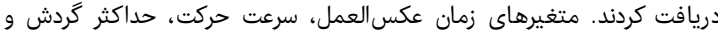

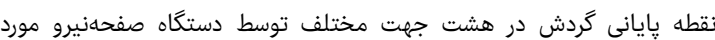
ارزيابى قرار گَرفت. تحليل دادهها توسط آزمون آنكووا و بهكمك نرمافزار

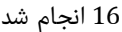
يافتهها: بعد از مداخلات درمانى، تمرينات تركيبى باعث بهببود بيشتر در زمان

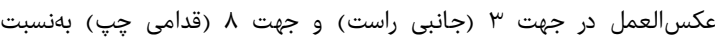

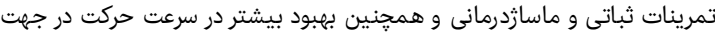

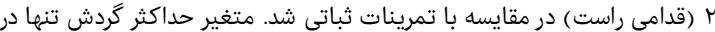

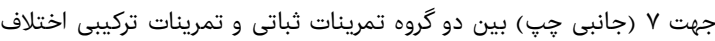

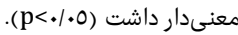

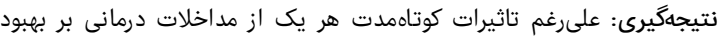

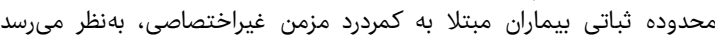

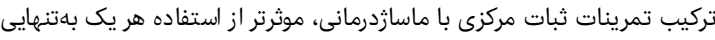

كليدوازمها: ماسار، تمرينات ثبات مركزى، محدوده ثباتى، كمردرد مزمن غيراختصاصى

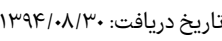

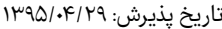
نويسنده مسئول: alishakeri310@gmail.com

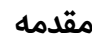

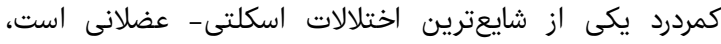

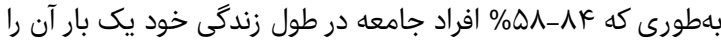

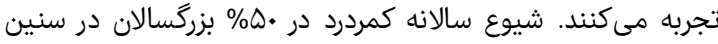

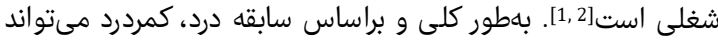

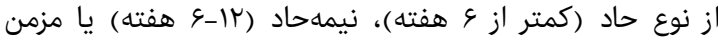

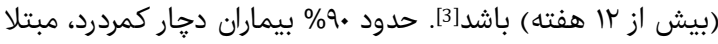

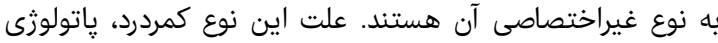

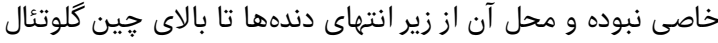

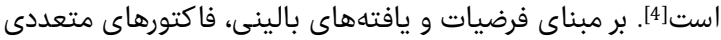

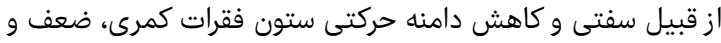

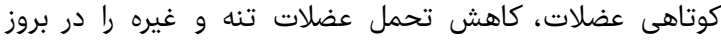
كمردرد دخيل دانستهاند[5].

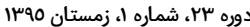




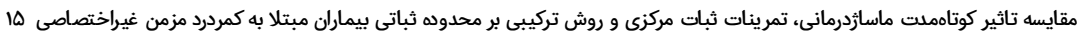

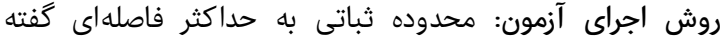

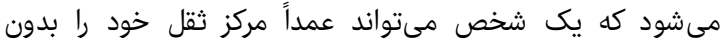

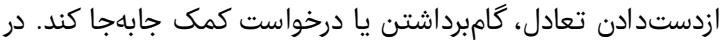

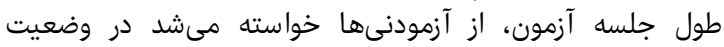

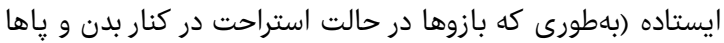

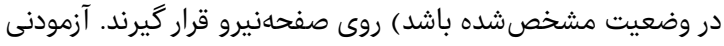

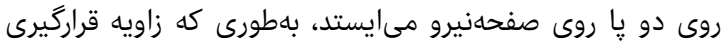

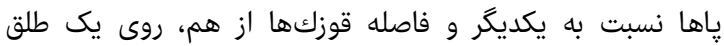

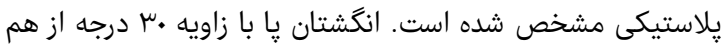

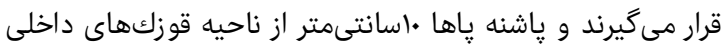

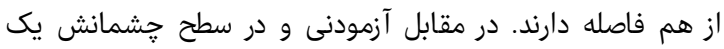

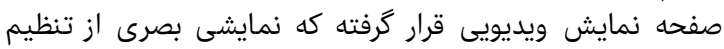

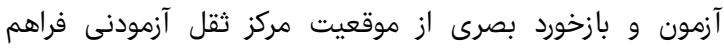

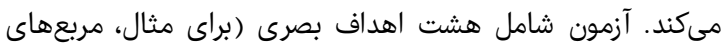

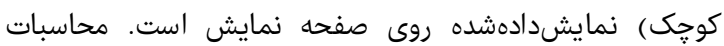

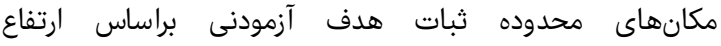

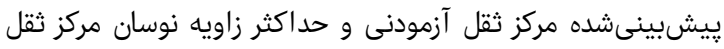

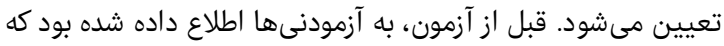

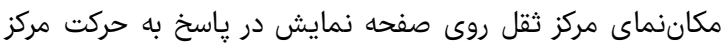

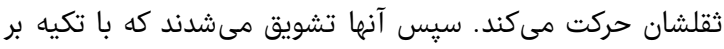

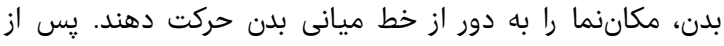

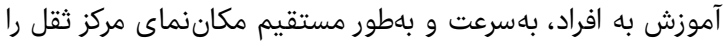

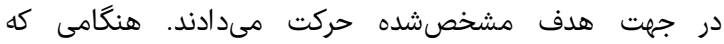

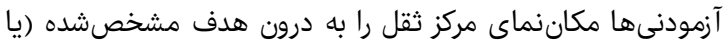

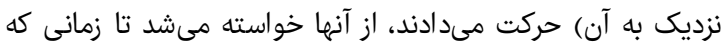

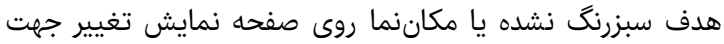

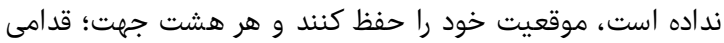

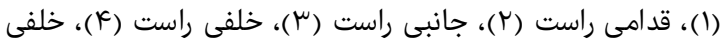

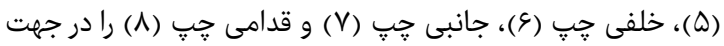

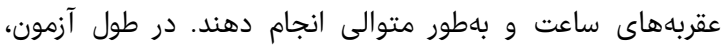

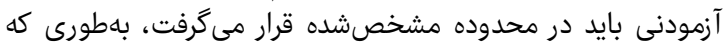

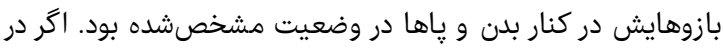

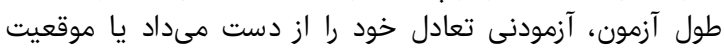

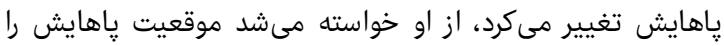

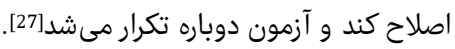

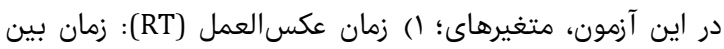

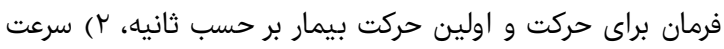

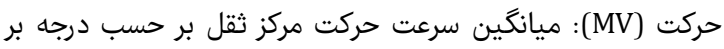
ثانيه،

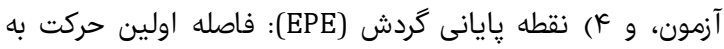

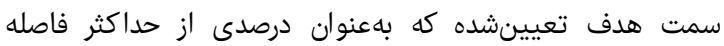

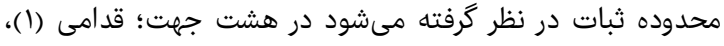

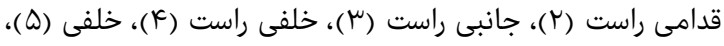

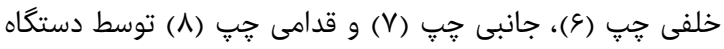

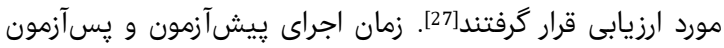

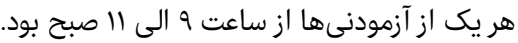

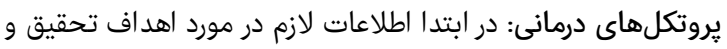

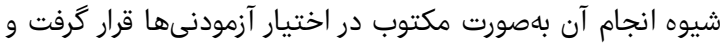

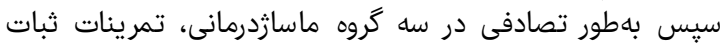

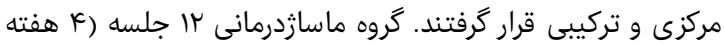

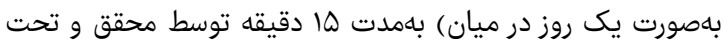

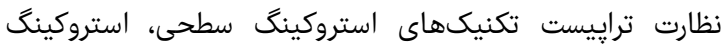

از طرفى شواهد استفاده از تمرينات ثباتى در درمان كمردرد مزمن كاني

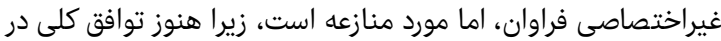

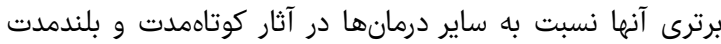

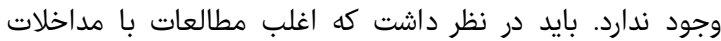

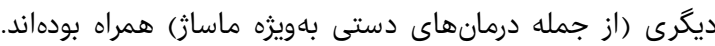

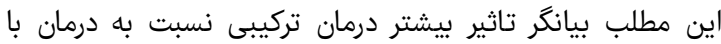

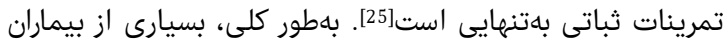

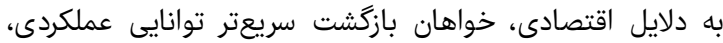

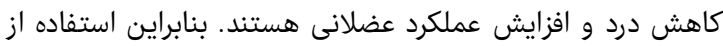

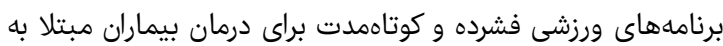

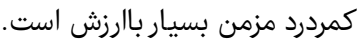

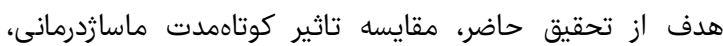

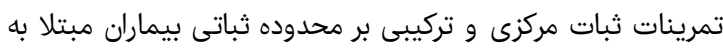
كمردرد مزمن غيراختصاصى بودي

\section{مواد و روشها}

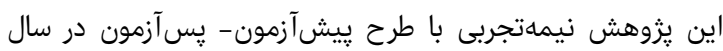

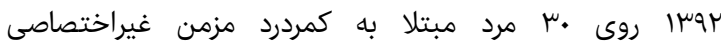

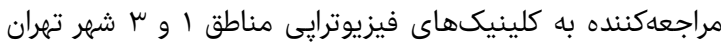

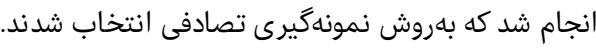

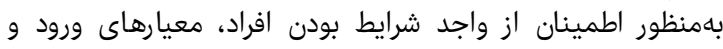

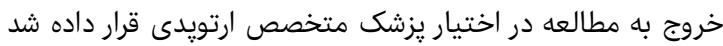

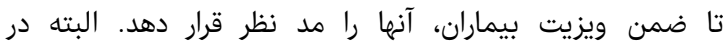

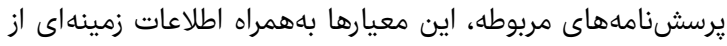

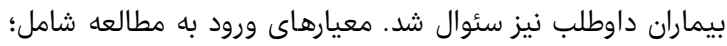

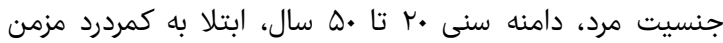

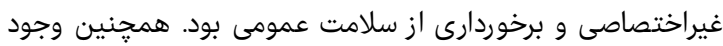

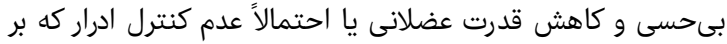

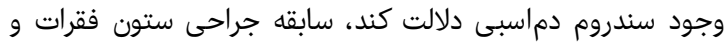

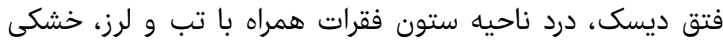

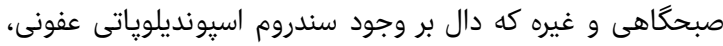
بدخيمى يا بيمارى التهابى باشد، وجود شكستخى فشارى ناشي ناشى از

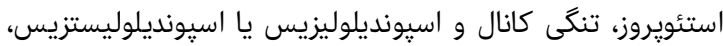

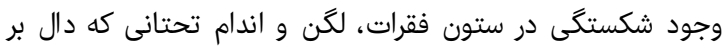

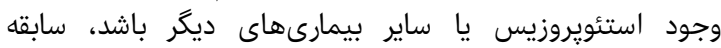

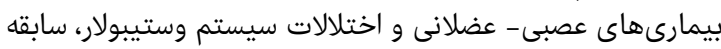

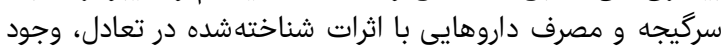

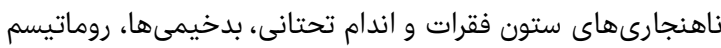

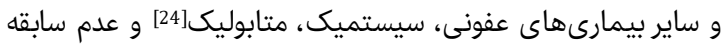

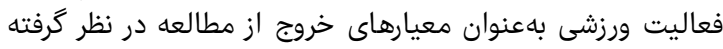

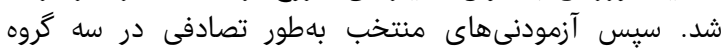

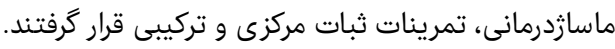

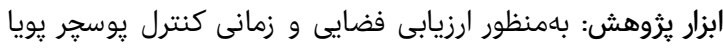

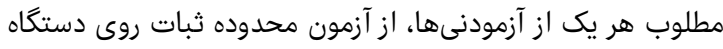

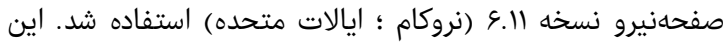

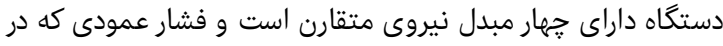

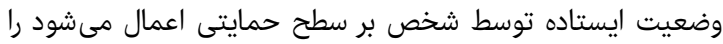

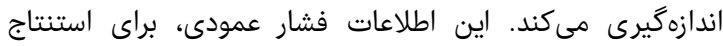

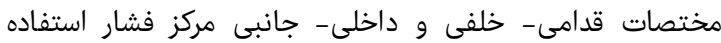

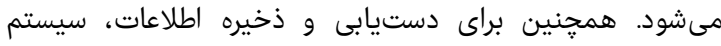
صفحهنيرو به يك كامييوتر متصل مى شود [26]. 


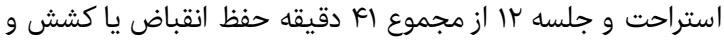

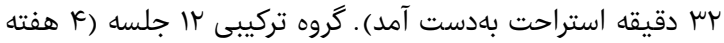

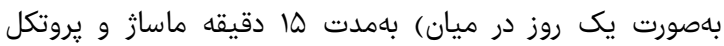

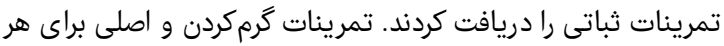

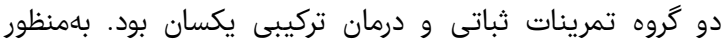

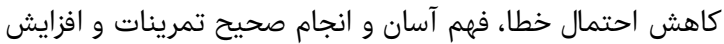

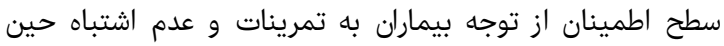

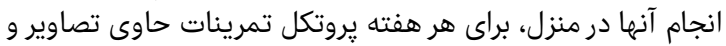

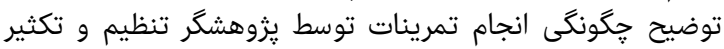

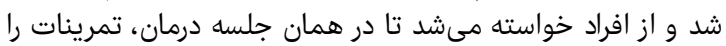

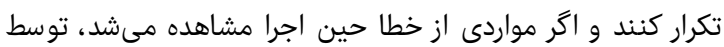

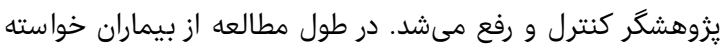

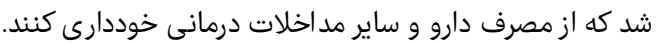

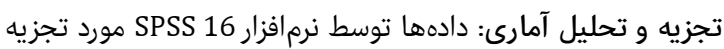

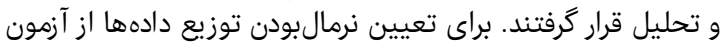

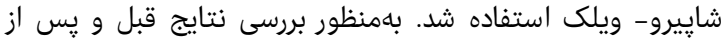

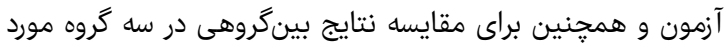
مطالعه، آزمون آنكووا مورد استفاده قرار گرقائ.

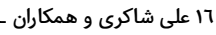

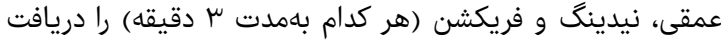

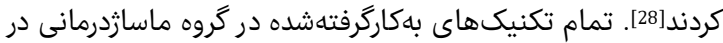

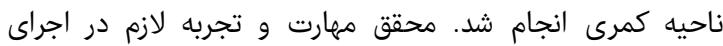

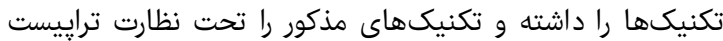
انجام داد.

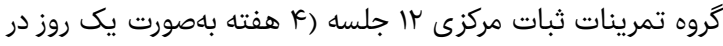

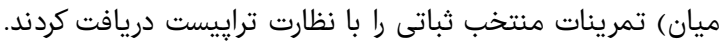

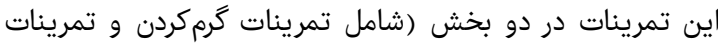

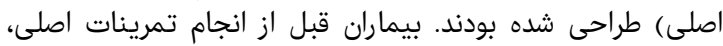

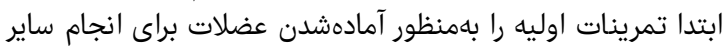

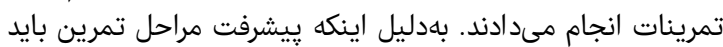

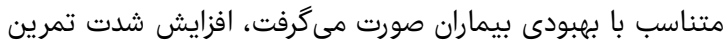

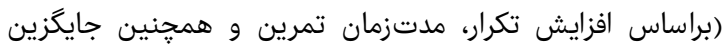

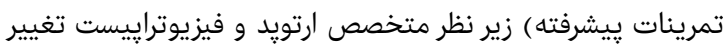

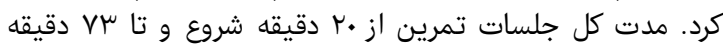

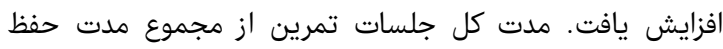

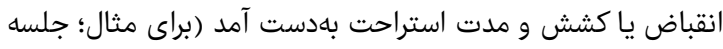

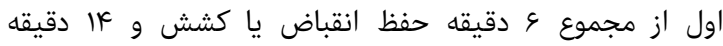

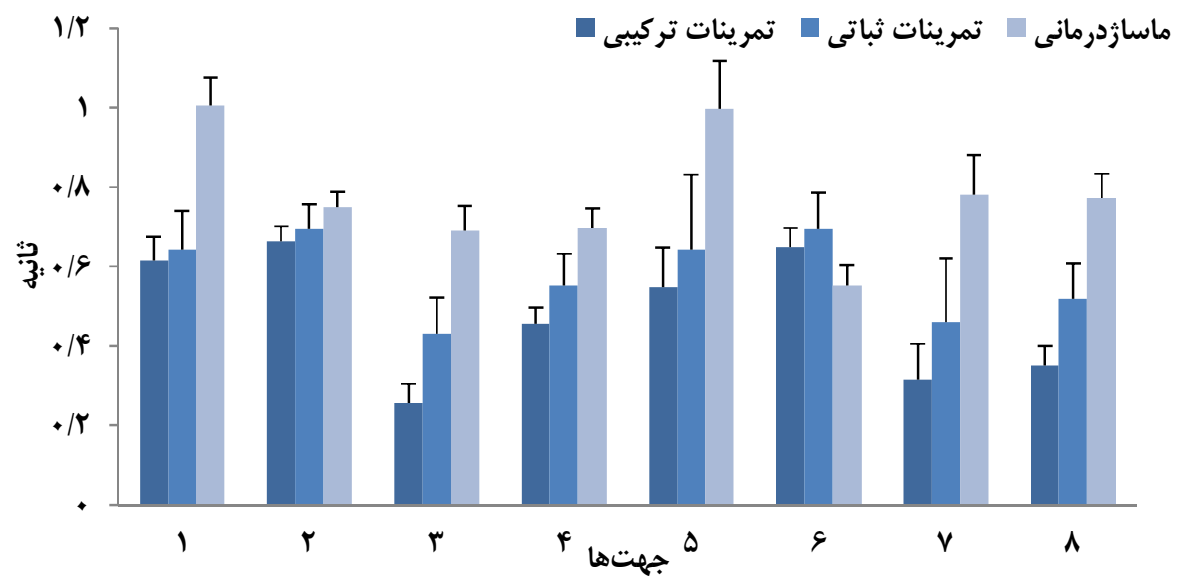

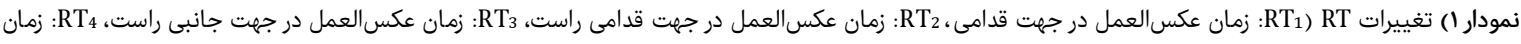

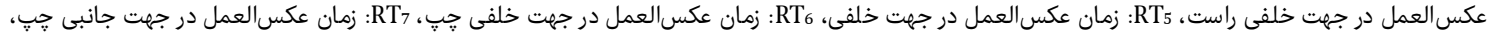

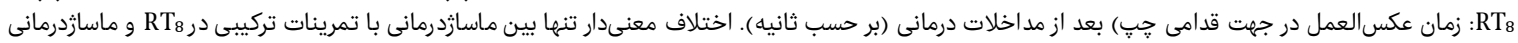

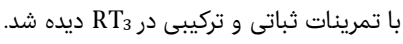

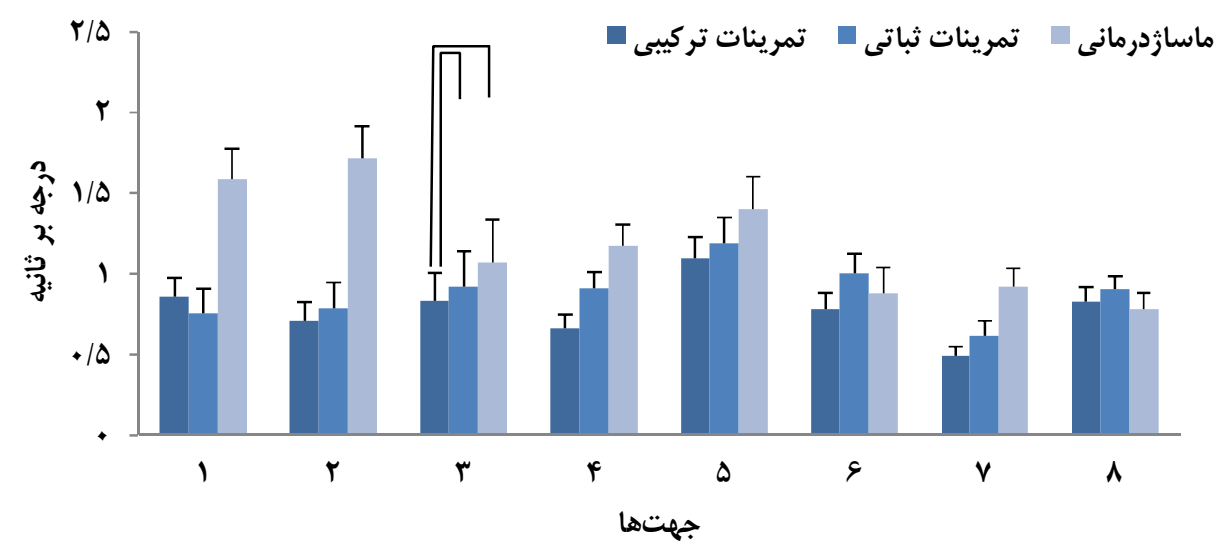

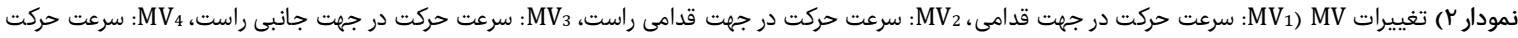

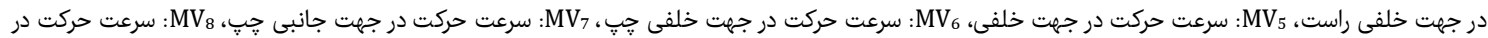

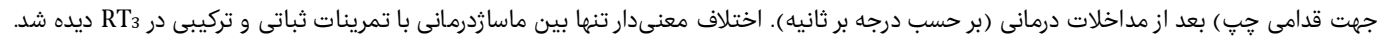




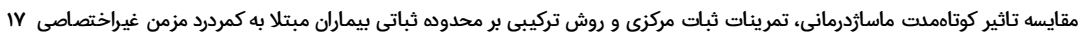

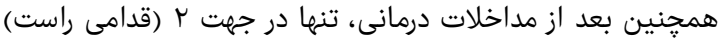

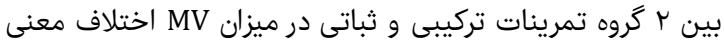

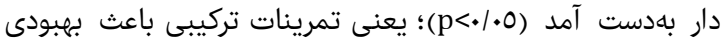

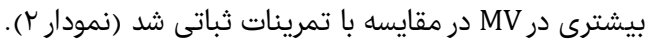
بعد از مداخلات درمانى، تنها دريات در جهت

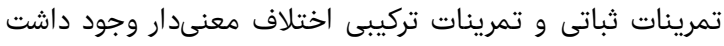

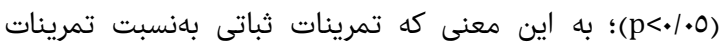

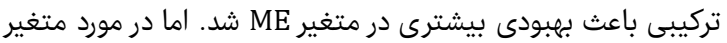

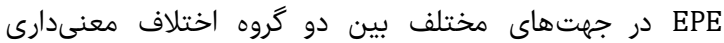

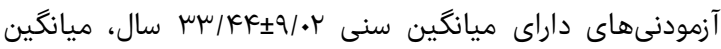

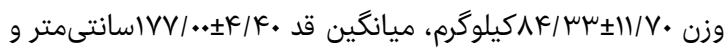

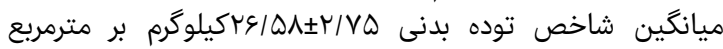
بودند.

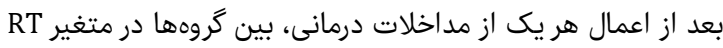

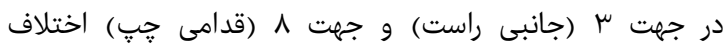

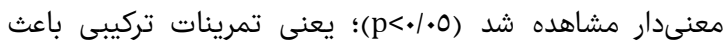

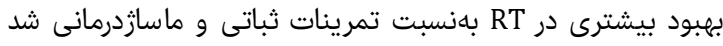

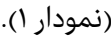
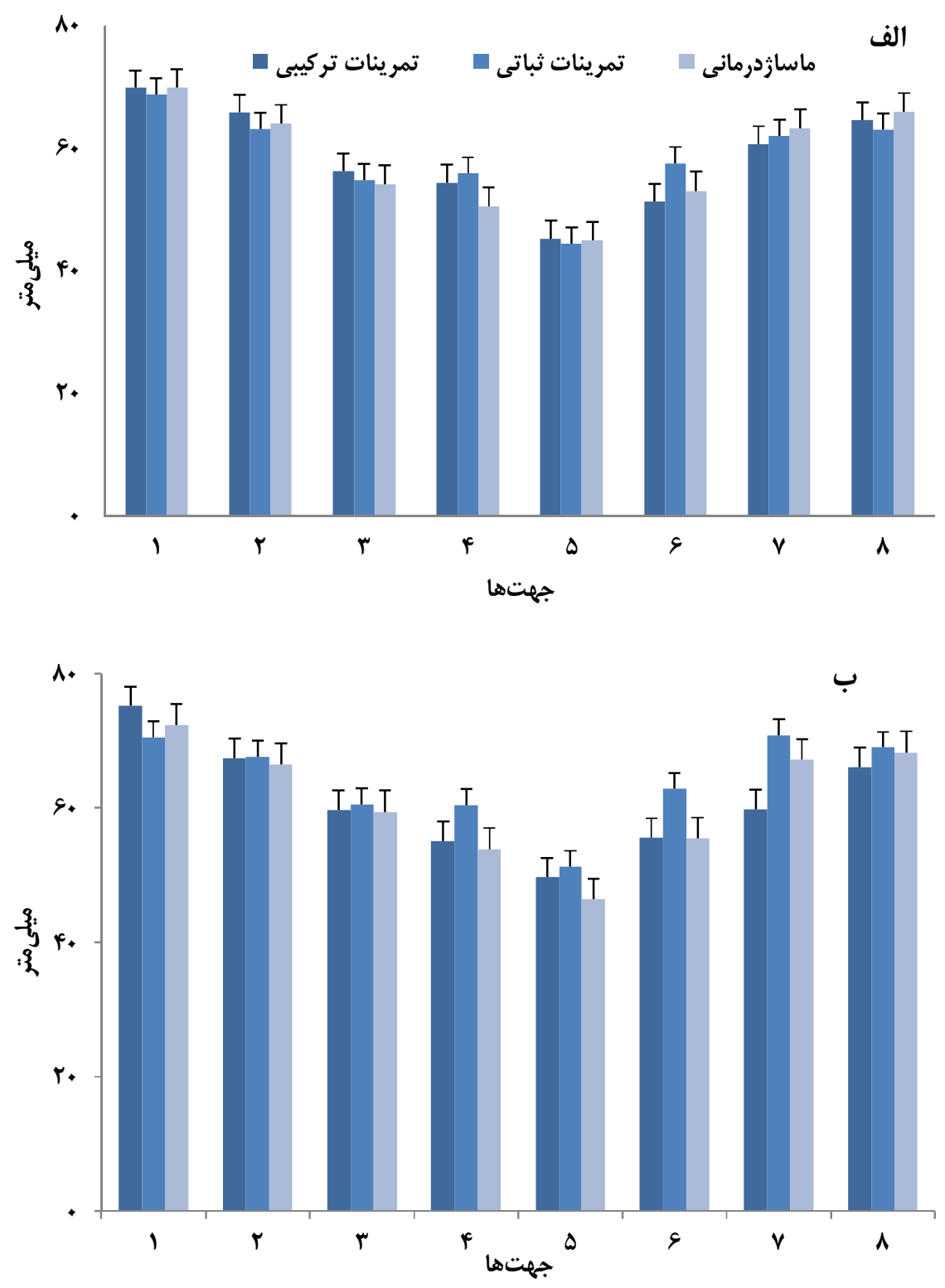

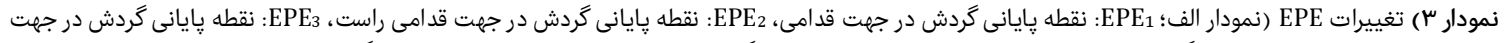

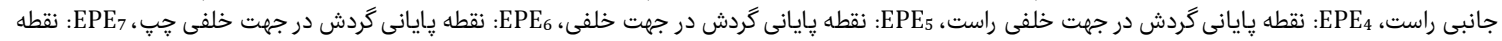

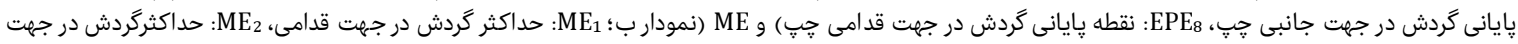
قدامى راست،

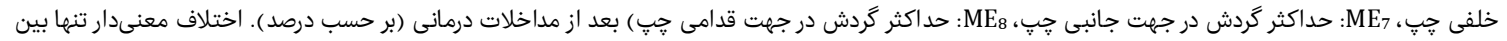
تمرينات ثباتى با تركيبى در در دئ ديده شد. 
آموزش و اجراى تمرينات ثباتى فشرده و تحت نظر بر شاخصهاى

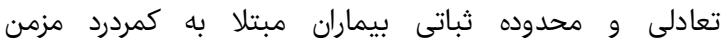

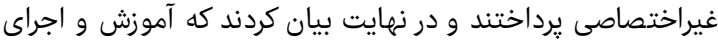

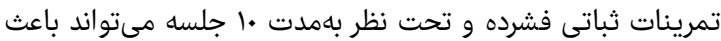

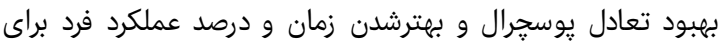

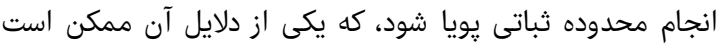

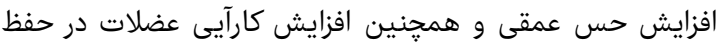

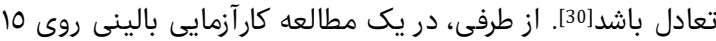

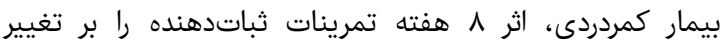

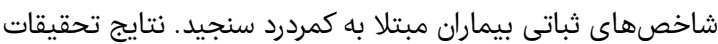

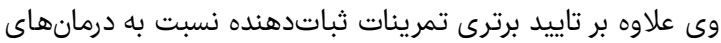

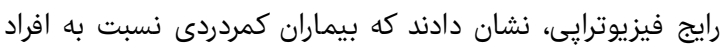

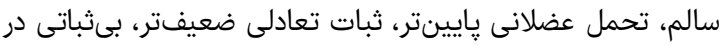

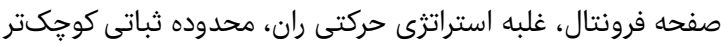

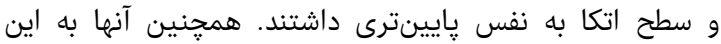

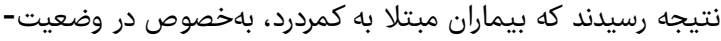

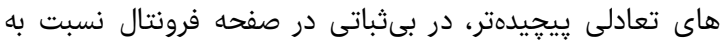

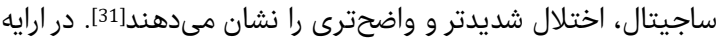

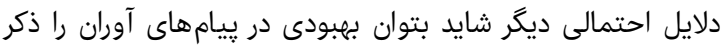

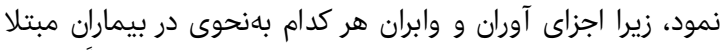

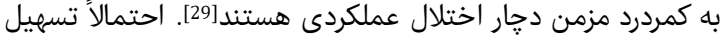
حس عمقى و همجنين افزايش كارآيى عضلات كمر و ران بهدردي هنبال

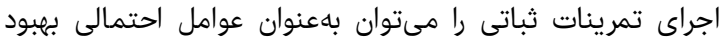
متغيرهاى EPE و ME

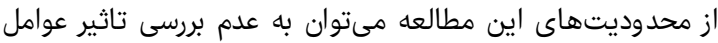

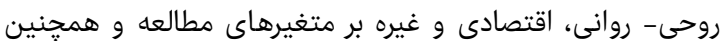

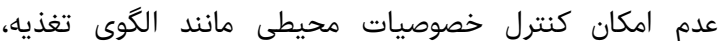

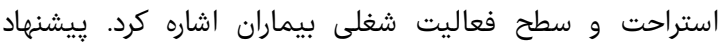

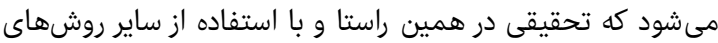

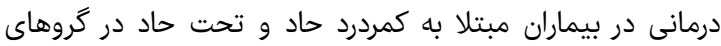

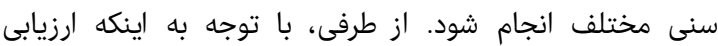

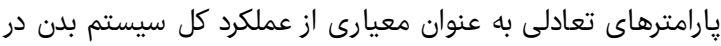

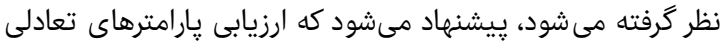
در اين بيماران مورد توجه قرار گيرد.

\section{نتيجهكيرى}

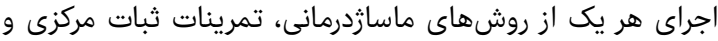

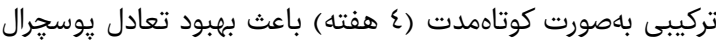

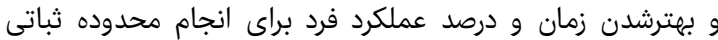

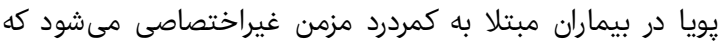

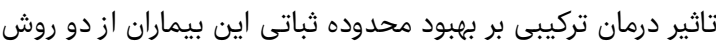

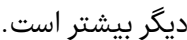

تشكر و قدردانى: از كليه افراد شركتكننده در اين تحقيق صميمانه

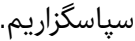

تاييديه اخلاقى: نويسندكان، كليه موارد اخلاقى مربوط به تحقيقات

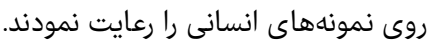

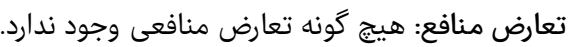

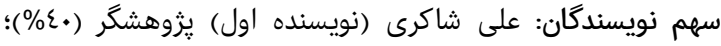

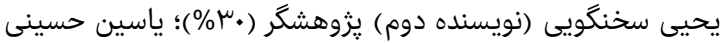

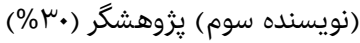

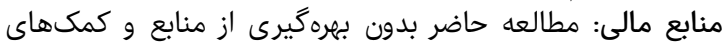

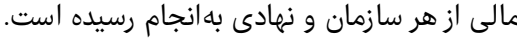

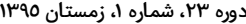

هدف از تحقيق حاضر مقايسه سه روش دوث درمانى ماساز، تمرينات

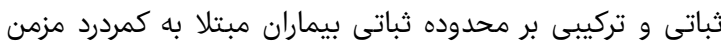

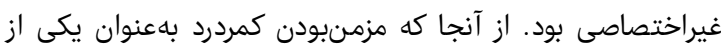

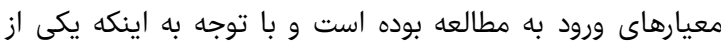

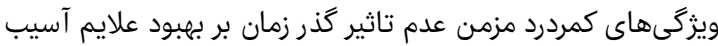

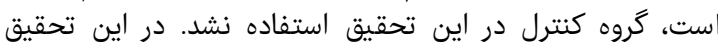

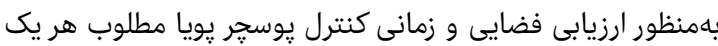

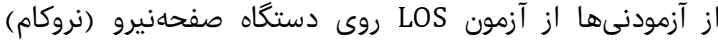

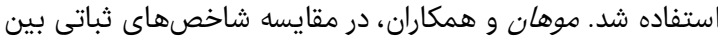

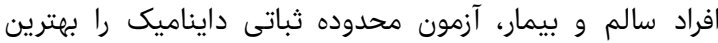

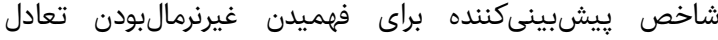

دانستند[29].

در مطالعه حاضر متغيرهاى RT، RT ، ME و ME و ME توسط دستكًاه،

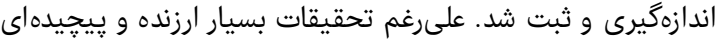

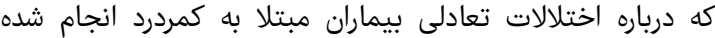

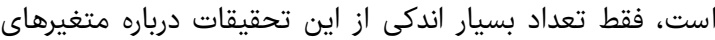

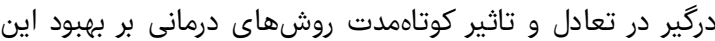

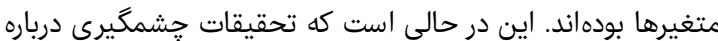

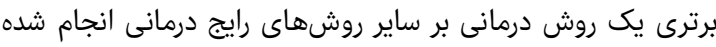

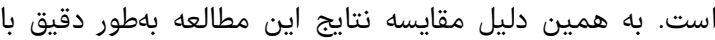
ساير نتايج مطالعات كذشته ميسر نيست دمايس نتايج

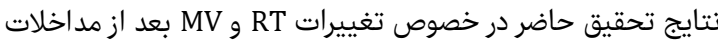
درمانى، ذشاندهنده تاثير بيشتر درمان تركيبى درئ نسبت به به تمرينات

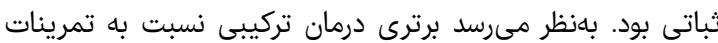

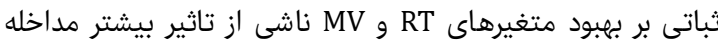

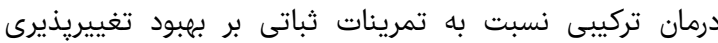

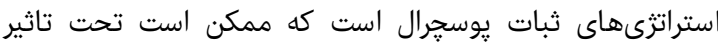

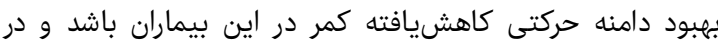

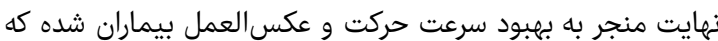
اين مساله ناشى از تاثيرات همزمان ماساز و تمرينات ثباتى بودي لوده

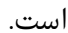

نتايج مطالعه حاضر در راستاى نتايج مرور نظاممند مى و جانسون

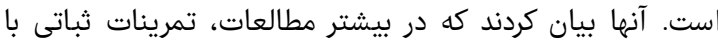

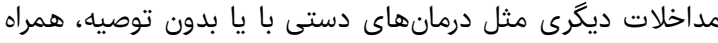

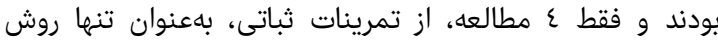

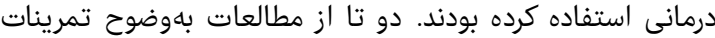

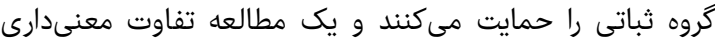

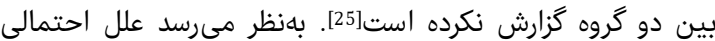

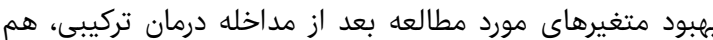

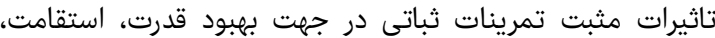

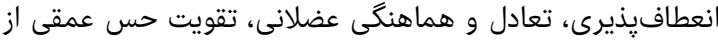

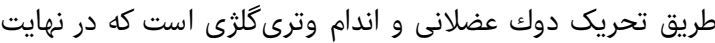

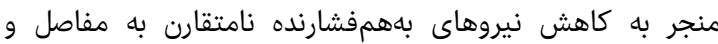

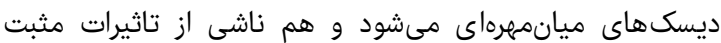

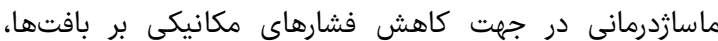

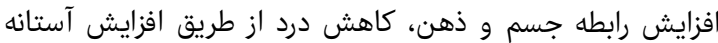

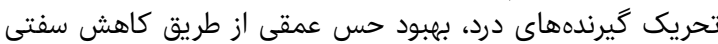
و اسياسم عضلانى است. تحرن.

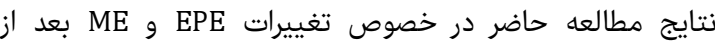

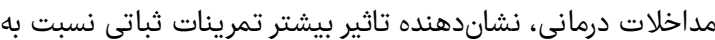

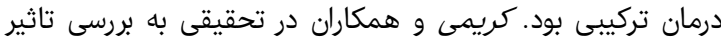

فصل نامه علمى - يُزوهشى افق دانش 
19 مقايسه تاثير كوتامدات ماساثدرمانى، تمرينات ثبات مركزى و روش تركيبى بر محدوده ثباتى بيماران مبتلا به كمردرد مزمن غيراختصاصى

and back problems. In: Korr IM, editor. The neurologic mechanisms in manipulative therapy. New York: Plenum; 1978. pp. 27-41.

18- Byrne K, Doody C, Hurly DA. Exercise therapy for low back pain: Asmall-scale exploratory survey of current physiotherapy practice in the Republic of Irland acute hospital setting. Man Ther. 2006;11(4):272-8.

19- Ebenbichler GR, Oddson LI, Kollmitzer J, Erim Z. Sensory-motor control of the lower back: Implications for rehabilitation. Med Sci Sports Exerc. 2001;33(11):1889-98.

20- Kofotolis N, Kellis E. Effects of two 4-week proprioceptive neuromuscular facilitation programs on muscle endurance, flexibility, and functional performance in women with chronic low back pain. Phys Ther. 2006; 86(7):1001-12.

21- Hubley-Kozey CL, Vezina MJ. Muscle activation during exercises to improve trunk stability in men with low backpain. Arch Phys Med Rehabil. 2002;83(8):1100-8. 22- Tsao H, Hodges PW. Persistence of improvements in postural strategies following motor control training in people with recurrent low back pain. J Electromyogr Kinesiol. 2008;18(4):559-67

23- Tsao H, Druitt TR, Schollum TM, Hodges PW. Motor training of the lumbar paraspinal muscles induces immediate changes in motor coordination in patients with recurrent low back pain. J Pain. 2010;11(11):1120-8. 24- Karimi N, Ebrahimi I, Ezzati K, Kahrizi S, Torkaman G, Arab AM. The effects of consecutive supervised stability training on postural balance in patients with chronic low back pain. Pak J Med Sci. 2009;25(2):177-81.

25- May S, Johnson R. Stabilisation exercises for low back pain: A systematic review. Physiotherapy. 2008;94(3):179-89.

26- Clark S, Rose DJ. Evaluation of dynamic balance among community-dwelling older adult fallers: A generalizability study of the Limits of Stability Test. Arch Phys Med Rehabil. 2001;82(4):468-74.

27- Clark S, Rose DJ, Fujimoto K. Generalizability of the limits of stability test in the evaluation of dynamic balance among older adults. Arch Phys Med Rehabil. 1997;78(10):1078-84.

28- Panahi F, Kamali Sarvestani F. Compared massage and modalities in the treatment of patients with nonspecific low back pain subacute and chronic. J Kermanshah Univ Med Sci. 2011;15(5):327-32. [Persian] 29- Mohan G, Pal PK, Sendhil KR, Thennarasu K, Usha BR. Quantitative evaluation of balance in patients with spinocerebellar ataxia type 1: A case control study. Parkinsonism Relat Disord. 2009;15(6):435-9.

30- Karimi N, Ebrahimi I, Ezzati K, Kahrizi S, Torkaman G, Arab AM. The effects of stability exercise on index stability and limits of stability in patients with mechanical chronic low back pain. Iran Rehabil J. 2012;12(2):21.

31- Radebold A, Cholewicki J, Polzhofer GK, Greene HS. Impaired postural control of the lumbar spine is associated with delayed muscle response times in patients with chronic idiopathic low back pain. Spine. 2001;26(7):724-30.

1- Robin DI. Epidemiology and risk factors for spine pain. Neurol Clin. 2007;25(2):353-71.

2- Walker BF, Muller R, Grant WD. Low back pain in Australian adults. health provider utilization and care seeking. J Manipulative Physiol Ther. 2004;27(5):327-35. 3- Stankovic A, Lazovic M, Kocic M, Zlatanovicd. Spinal segmental stabilization exercises combined with traditional strengthening exercise program in patients with chronic low back pain. Acta Fac Med Naiss. 2008;25(3):165-70.

4- Furlan $\mathrm{AD}$, Imamura M, Dryden T, Irvin E. Massage for low back pain: An updated systematic review within the framework of the Cochrane Back Review Group. Spine (Phila Pa 1976). 2009;34(16):1669-84.

5- Clare H, Adams R, Maher C. A systematic review of efficacy of McKenzie therapy for spinal pain. Aust J Physiother. 2004;50(4):209-16.

6- Karimi N, Ebrahimi E, Torkaman G, Kahrizi S, Maghsoudi Pour M, Ezzati K. The effects of stability training on postural indices and limit of stability in male patients with nonspecific chronic low back pain. Phys Treat J. 2012;2(1):31-8. [Persian]

7- Kapandji IA. Physiology of the joint. 2nd edition. Edinburgh London: Churchill Livingston; 1982. pp. 68102.

8- Kisner C. Therapeutic exercise: Foundations and techniques. 2nd edition. Philadelphia: F.A. Davis; 1990. pp. 222-54.

9- Karimi N, Ebrahimi I, Kahrizi S, Torkaman G. Evaluation of postural balance using the biodex balance system in subjects with and without low back pain. Pak J Med Sci. 2008;24(3):1-6.

10- Kendall FP, McCreary EK, Provance PG. Muscle testing and function. $4^{\text {th }}$ edition. Philadelphia: Lippincott Williams \& Wilkins; 1993. pp. 321-65.

11- Langevin HM, Sherman KJ. Pathophysiological model for chronic low back pain integrating connective tissue and nervous system mechanisms. Med Hypotheses. 2007;68(1):74-80.

12- Norris CM. Spinal stabilisation: 4. Muscle imbalance and the low back. Physiotherapy. 1995;81(3):127-38.

13- Akuthota V, Ferreiro A, Moore T, Fredericson M. Core stability exercise principles. Curr Sports Med Rep. 2008;7(1):39-44.

14- Richardson C, Jull G, Toppenberg R, Comerford M. Techniques for active lumbar stabilisation for spinal protection: a pilot study. Aust J Physiother. 1992;38(2):105-12.

15- O'Sullivan, P. Diagnosis and classification of chronic low back pain disorders: maladaptive movement and motor control impairments as underlying mechanism. Man Ther. 2005;10(4):242-55.

16- Janda V. Muscle strength in relation to muscle length, pain and muscle imbalance. In: Harms-Ringdahl $\mathrm{K}$, editor. Muscle strength (International Perspectives in Physical Therapy). Churchill Livingston, Edinburgh: W.B. Saunders Company; 1993. pp. 112-325.

17- Janda V. Muscles, central nervous motor regulation 